\title{
Dynamic contrast-enhanced MRI as a predictor of programmed death ligand-1 expression in patients with oral squamous cell carcinoma
}

\author{
NOUHA TEKIKI $^{1}$, MARIKO FUJITA $^{1}$, TATSUO OKUI ${ }^{2,3}$, HOTAKA KAWAI $^{4}$, MAY WATHONE OO $^{4}$, \\ TOSHIYUKI KAWAZU ${ }^{1}$, MIKI HISATOMI ${ }^{5}$, SHUNSUKE OKADA ${ }^{5}$, YOHEI TAKESHITA ${ }^{1}$, MAJD BARHAM ${ }^{1}$, \\ HITOSHI NAGATSUKA ${ }^{4}$, YOSHINOBU YANAGI ${ }^{6}$ and JUN-ICHI ASAUMI $^{1,5}$ \\ Departments of ${ }^{1}$ Oral and Maxillofacial Radiology; ${ }^{2}$ Oral and Maxillofacial Surgery, \\ Okayama University Graduate School of Medicine, Dentistry and Pharmaceutical Sciences, \\ Okayama 700-8558; ${ }^{3}$ Department of Oral and Maxillofacial Surgery, Shimane University Faculty of Medicine, \\ Izumo, Shimane 693-8501; ${ }^{4}$ Department of Oral Pathology and Medicine, Okayama University Graduate School of \\ Medicine, Dentistry and Pharmaceutical Sciences; ${ }^{5}$ Department of Oral Diagnosis and Dentomaxillofacial Radiology, \\ Okayama University Hospital; ${ }^{6}$ Department of Dental Informatics, Okayama University Graduate School of \\ Medicine, Dentistry and Pharmaceutical Sciences, Okayama 700-8558, Japan
}

Received April 23, 2021; Accepted August 11, 2021

DOI: 10.3892/ol.2021.13039

\begin{abstract}
Immune checkpoint inhibitors (ICIs) targeting programmed death ligand-1 (PD-L1) are highly promising therapies for oral squamous cell carcinoma (OSCC). The assessment of PD-L1 expression may help predicting the therapeutic effect of ICIs and, thus, benefit patient selection. Contrast index $(\mathrm{CI})$ parameters derived from dynamic contrast-enhanced magnetic resonance imaging (DCE-MRI) have been proven as efficient to assess microvessel density (MVD) in OSCC. The present study aimed to determine the correlation between DCE-MRI parameters and MVD and between DCE-MRI parameters and PD-L1 expression to determine whether DCE-MRI could be used non-invasively to evaluate PD-L1 expression in patients with OSCC. A total of 21 patients with primary OSCC who had undergone a 3T MRI scan, including DCE-MRI, were included in the present study, and $\mathrm{CI}$ curve-derived parameters were examined. The MVD and PD-L1 expression in the surgically resected specimens were analyzed using immunohistochemistry (IHC) staining for CD31 and IHC staining for PD-L1, respectively. The results demonstrated that the expression levels of these markers
\end{abstract}

Correspondence to: Dr Mariko Fujita, Department of Oral and Maxillofacial Radiology, Okayama University Graduate School of Medicine, Dentistry and Pharmaceutical Sciences, 2-5-1 Shikata-cho, Kita-ku, Okayama 700-8558, Japan

E-mail: gmd421082@s.okayama-u.ac.jp

Key words: head and neck cancer, oral squamous cell carcinoma, dynamic contrast-enhanced magnetic resonance imaging, microvessel density, programmed death ligand-1, immunotherapy were correlated with DCE-MRI parameters. PD-L1 expression levels were found to be significantly correlated with the maximum CI (CI-max; P=0.007), peak CI (CI-peak; $\mathrm{P}=0.007)$, maximum $\mathrm{CI}$ gain $(\mathrm{CI}$-gain; $\mathrm{P}=0.006)$ and $\mathrm{MVD}(\mathrm{P}=0.001)$ values. The mean CI-max, CI-peak, CI-gain and MVD values were significantly higher in tumors with high PD-L1 expression $(\mathrm{P}<0.05)$. MVD levels were also significantly correlated with the time of CI-max (T-max; $\mathrm{P}=0.003)$ and CI-gain $(\mathrm{P}=0.037)$. The mean CI-gain was significantly increased, and the mean T-max was significantly shorter in high MVD tumors $(\mathrm{P}<0.05$ and $\mathrm{P}<0.01$, respectively). In summary, the findings from the present study confirmed the correlation between CI parameters, derived from DCE-MRI, and MVD, and suggested that these parameters may be correlated with PD-L1 expression in OSCC tumor cells.

\section{Introduction}

The use of immunotherapy has revolutionized the treatment of numerous types of cancer, such as lung, gastric and cervical cancers $(1,2)$. New indications, treatment strategies and thus, consensus recommendations, continuously emerge for immune checkpoint inhibitors (ICIs) designed for different malignancies $(3,4)$. The ICIs nivolumab (Opdivo; Bristol Myers Squibb) and pembrolizumab (Keytruda; Merck Sharpe $\&$ Dohme-Hoddesdon) have been approved in the recent years for the treatment of patients with recurrent/metastatic head and neck squamous cell carcinoma (HNSCC) and who experience disease progression after treatment with a platinum-based chemotherapy agent (3-5). These novel drugs aim to target an important immunological checkpoint in HNSCC and oral squamous cell carcinoma (OSCC), which is the programmed death-1 receptor (PD-1)/programmed death ligand-1 (PD-L1) interaction $(5,6)$. Nivolumab and pembrolizumab block 
the inhibitory interaction between PD-1 and PD-L1, which reactivates the immune system and enhances tumor cell elimination. ICIs targeting PD-1/PD-L1 have demonstrated clinical efficacy in HNSCC, highlighting the important role of ICIs in the management of the disease $(6,7)$. PD-L1 expression is commonly used as a biomarker to predict the therapeutic effect of and response rates to the immunotherapy drugs, nivolumab and pembrolizumab (8). In patients with recurrent/metastatic HNSCC, biopsies are commonly used for confirmation of disease recurrence, and it has been suggested that these biopsies should also be used to evaluate PD-L1 expression (8). However, biopsies are surgically invasive and place a physical burden on the patient. Therefore, a complementary and minimally invasive procedure for assessing PD-L1 expression may be beneficial for patients. Magnetic resonance imaging (MRI) is a widespread imaging technique that is frequently used for the diagnosis of HNSCC. In particular, dynamic contrast-enhanced (DCE)-MRI produces functional images and provides insight into the tumor microvasculature, which can help predict the outcome. Thus, DCE-MRI is currently used as a non-invasive method that provides measures related to immunohistochemical analyses $(9,10)$, which helps investigating numerous histopathological features that may predict tumor behavior. Microvessel density (MVD) is a key histopathological feature worthy of investigation, which has been reported to be a useful prognostic indicator in various types of malignant tumors $(11,12)$. Assessment of MVD involves the evaluation of vessels following staining with the endothelial cell marker CD31 (11). MVD reflects the intensity of angiogenesis within the tumor, which is reportedly associated with tumor growth or metastasis in OSCC $(9,12)$.

A previous study suggested that angiogenesis may influence the enhancement patterns on DCE-MRI scans, and that MVD is significantly associated with DCE-MRI parameters in patients with OSCC (9). MVD was also reported to be positively associated with PD-L1 expression in patients with classical Hodgkin's lymphoma, as the mean MVD of PD-L1-positive tumors was found to be slightly higher compared with that of PD-L1-negative tumors (11). Based on these studies, the present study hypothesized that DCE-MRI may be able to predict PD-L1 expression.

In our institution, DCE-MRI is performed as a preoperative examination for patients with HNSCC, particularly those with OSCC. Considering that DCE-MRI is a non-invasive method, the identification of valid DCE-MRI parameters for estimating the expression of PD-L1 and subsequently assessing the therapeutic effect of ICIs may reduce the physical burden that biopsies incur to patients and expand to its clinical application. To the best of our knowledge, no previous study has investigated the potential roles of DCE-MRI signal intensity (SI)- and contrast index (CI)-based parameters for predicting the therapeutic effect of ICIs in patients with OSCC.

\section{Materials and methods}

Patients. The study protocol was approved by the Institutional Review Board of Okayama University Graduate School of Medicine, Dentistry and Pharmaceutical Sciences, Okayama University Hospital, Ethics Committee (approval no. 1807-008). All patients provided written informed consent prior to participation. Between October 2012 and March 2017, 59 patients who underwent DCE-MRI at Okayama University Hospital (Okayama, Japan) were histopathologically diagnosed with primary OSCC. Patients with T4 tumors according to the Tumor-Node-Metastasis (TNM) classification (13) were excluded, as T4 tumors are large and likely to invade the peripheral tissue, which would produce an erroneous SI on a DCE-MRI scan. Of the selected patients with T1, T2 and T3 tumors, patients were excluded if the lesions were too small for the SI to be calculated, if they had substantial metal-induced artifacts, if the tumor exhibited areas of necrosis, or if patient movement was recorded during the scan. Furthermore, patients who underwent DCE-MRI using a different processing platform (Tissue 4D; Siemens Healthineers), which included 30 scans, and patients whose DCE-MRI scans were acquired in the coronal plane, were also excluded. Thus, according to the inclusion criteria, 21 patients (12 men and 9 women; age range, 34-87 years; mean age, 64 years) were included as the final patient cohort in the present study. The distribution of the primary lesions was as follows: Tongue, $n=8$; maxillary gingiva, $n=3$; mandibular gingiva, $n=3$; floor of the mouth, $\mathrm{n}=4$; buccal mucosa, $\mathrm{n}=2$; and palate, $\mathrm{n}=1$.

MRI protocol. The MRI examinations were performed using a 3T unit (MAGNETOM Skyra; Siemens Healthineers) with a head coil or head and neck coil. T1-weighted images (T1WIs) or T1-weighted fluid-attenuated inversion recovery (T1-FLAIR) images were acquired with a spin-echo sequence, and short inversion time inversion recovery (STIR) images were acquired for all cases with a turbo spin-echo sequence. Images were taken in both the axial and coronal planes. The section level for the dynamic study was then selected from the acquired T1WIs and STIR images. DCE-MRI in the selected section was performed using three-dimensional fast imaging with a steady-state precession sequence. The imaging parameters of the dynamic study were as follows: Repetition time, 4.53-7.48 msec; echo time, 1.7-3.06 msec; and flip angle, $12^{\circ}$. The first DCE-MRI series was composed of 14 consecutive scans, the acquisition time for each scan was $14 \mathrm{sec}$ and the interscan interval was $1 \mathrm{sec}$, resulting in a total scan time of $210 \mathrm{sec}$. Before the second scan, $0.2 \mathrm{ml} / \mathrm{kg}$ gadopentetate dimeglumine (Magnevist ${ }^{\circledR}$; Nihon Schering) was administered intravenously for $6 \mathrm{sec}$ at a rate of $\sim 2.0 \mathrm{ml} / \mathrm{sec}$ via manual injection. Contrast-enhanced (CE) T1WIs with fat suppression or CE T1-FLAIR images with fat suppression were acquired after DCE-MRI.

Evaluation of DCE-MRI parameters. For each lesion, the region of interest (ROI) was drawn on the DCE images to include the region containing the greatest diameter of the tumor (Fig. 1A). The SI of each ROI was calculated using a workstation (Synapse Vincent; Fujifilm Medical Co.). The CI was calculated using the following formula: [SI (post-contrast)-SI (pre-contrast)]/SI (pre-contrast). The time course of the CI was then plotted to obtain a CI curve. Using the CI curve, the following DCE-MRI parameters were defined: Maximum CI (CI-max; the maximum amplitude of contrast enhancement) and time of CI-max (T-max; the time at which CI-max occurred). The peak CI (CI-peak), which is the first CI measurement that satisfied CI-max x 0.90, and the 
A

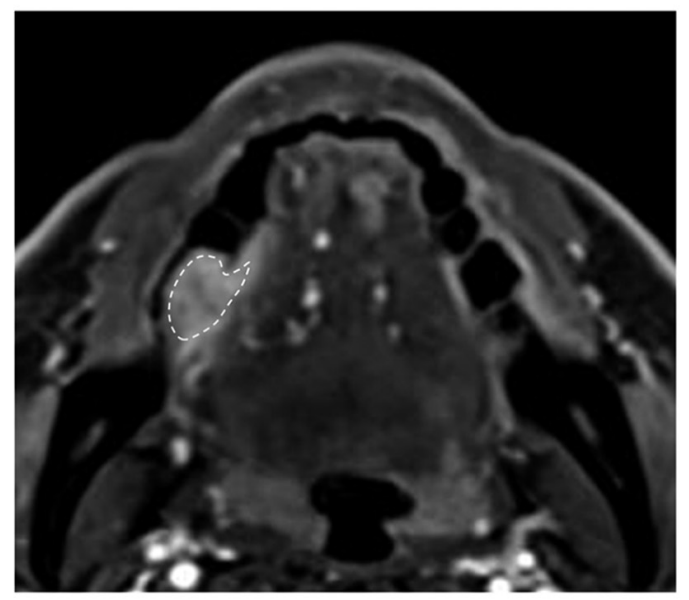

$\mathrm{B}$

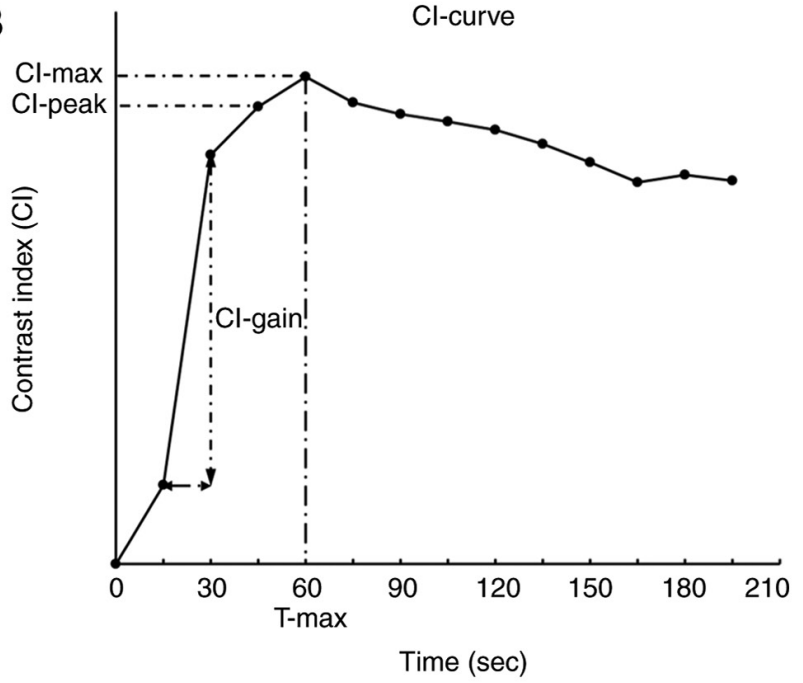

Figure. 1. DCE-MRI image and typical CI curve of OSCC. (A) Region of interest was drawn on the DCE-MRI image to include the region containing the greatest diameter of the tumor. (B) CI curve was constructed using DCE-MRI and CI curve parameters. DCE-MRI, dynamic contrast-enhanced magnetic resonance imaging; CI, contrast index.

maximum CI gain (CI-gain), which is the maximum gradient on the upslope phase of the enhancement curve that indicates the difference in the CI between two consecutive images, were calculated (Fig. 1B).

Preparation of surgically resected specimens for histological examinations. The surgically resected specimens were fixed in formalin and embedded in paraffin using a standardized procedure (14). The representative tumor sections were selected so that the longest diameter in the plane of measurement containing the largest amount of tumor would be included. The representative tumor sections were cut into $3-\mu \mathrm{m}$ serial sections and mounted on APS-coated glass slides. These sections were subsequently used for immunohistochemistry (IHC).

IHC. Paraffin-embedded tissue sections were deparaffinized in a series of xylene for $15 \mathrm{~min}$, rehydrated through a decreasing series of graded ethanol (100, 90, 80 and 70\%), incubated in $3 \%$ hydrogen peroxide/methanol solution for $30 \mathrm{~min}$ at room temperature to quench the endogenous peroxidase activity, and washed with distilled water for $5 \mathrm{~min}$. For CD31 detection, antigen retrieval was performed by heating sections with $0.01 \mathrm{~mol} / \mathrm{l}$ citrate buffer $\left(\mathrm{pH} \mathrm{9.0)}\right.$ at $120^{\circ} \mathrm{C}$ for $15 \mathrm{~min}$ in a pressure cooker. For PD-L1 and vascular endothelial growth factor (VEGF) detection, antigen retrieval was performed by heating sections with $0.01 \mathrm{~mol} / \mathrm{l}$ citrate buffer $(\mathrm{pH} 6.0)$ at $100^{\circ} \mathrm{C}$ for $3 \mathrm{~min}$ in a microwave. Following antigen retrieval, the sections were blocked with $10 \%$ normal goat serum (Vector Laboratories, Inc.) for $20 \mathrm{~min}$ at room temperature in a humidified chamber. The sections were subsequently incubated with the following primary antibodies at $4^{\circ} \mathrm{C}$ overnight: Anti-CD31 (mouse; 1:100; cat. no. CD31-607-L-CE; Leica Biosystems Nussloch GmbH), anti-PD-L1 (rabbit; 1:500; cat. no. ab205921; Abcam) and anti-VEGF (rabbit; 1:100; cat. no. ab183100; Abcam). Following primary antibody incubation, the sections were incubated with a secondary biotinylated antibody (cat. no. PK-6101, Vector Laboratories, Inc.) using the avidin-biotin complex method. Color development was performed by incubating the sections with $0.02 \%$ Histofine ${ }^{\circledR}$ DAB substrate (Nichirei Biosciences, Inc.) at room temperature prior to counterstaining with Mayer's hematoxylin. Stained sections were visualized under an optical microscope at low (x20) and high (x100 and x200) magnification.

MVD quantification. MVD in vascular hotspots was quantified according to the consensus guidelines for the use and interpretation of angiogenesis assays and Weidner's method $(15,16)$. Vascular hotspots are defined as areas with a high concentration of new, but inefficient blood vessels, which have sprouted from existing vessels (15). Weidner's method states that any positively stained individual endothelial cell or endothelial cell cluster that is clearly separate from adjacent microvessels, tumor cells and other connective tissue elements, should be considered as a single, countable microvessel. The presence of vessel lumens is not necessary for a structure to be defined as a microvessel $(15,16)$. The cancer and stroma areas in the specimens were identified according to specific morphological features, including color, texture and contextual features (17), in addition to observing respective H\&E-stained slides. Slides were first examined at a low magnification (x20) to scan the entire stained tumor section and to identify vascular hotspots. Images of five vascular hotspots were then acquired at x200 magnification. CD31-positive microvessels were counted in the five acquired hotspot images and the mean MVD value, which has no units, was determined. Only cells that were morphologically compatible with the phenotype of endothelial cells based on their size and shape were counted as microvessels, to avoid the inclusion of macrophages. For each tumor, the MVD was defined as the number of microvessels in a microscopic field of $0.67 \mathrm{~mm}^{2}$. The samples were sorted into low and high MVD groups based on the median MVD.

Assessment of PD-L1 expression. To semi-quantify PD-L1 expression, the slides were first examined at a low magnification (x20). All areas in the tissue section were observed to appropriately evaluate the expression of PD-L1 on tumor cells. 
Table I. Clinicopathological characteristics, DCE-MRI parameters and immunohistochemical findings of patients with oral squamous cell carcinoma.

\begin{tabular}{|c|c|c|c|c|c|c|c|c|c|c|c|}
\hline Number & Age & Sex & Region & TNM & CI-max & T-max & CI-peak & CI-gain & MVD & VEGF & PD-L1 \\
\hline 1 & 65 & M & Maxilla & T1N0M0 & 1.00 & 43.60 & 0.90 & 0.57 & 49.25 & 89.90 & PD-L1 ${ }^{\text {+low }}$ \\
\hline 2 & 58 & M & Tongue & T1N0M0 & 1.61 & 89.90 & 1.45 & 0.82 & 24.80 & 71.70 & PD-L1 ${ }^{\text {+low }}$ \\
\hline 3 & 68 & M & Mouth floor & T1N0M0 & 1.17 & 59.70 & 1.05 & 1.02 & 73.80 & 58.30 & PD-L1 ${ }^{\text {thigh }}$ \\
\hline 4 & 55 & M & Tongue & T1N0M0 & 2.28 & 29.30 & 2.05 & 1.14 & 73.40 & 99.50 & PD-L1 ${ }^{\text {thigh }}$ \\
\hline 5 & 77 & $\mathrm{~F}$ & Mandibular & T2N0M0 & 1.04 & 87.20 & 0.93 & 0.59 & 42.00 & 93.80 & PD-L1' \\
\hline 6 & 65 & $\mathrm{~F}$ & Mandibular & T2N0M0 & 1.33 & 87.20 & 1.20 & 0.49 & 32.00 & 97.00 & PD-L1' \\
\hline 7 & 34 & $\mathrm{~F}$ & Tongue & T2N0M0 & 1.51 & 60.10 & 1.36 & 1.23 & 5.20 & 89.30 & PD-L1' \\
\hline 8 & 54 & M & Tongue & T2N0M0 & 1.29 & 75.00 & 1.16 & 0.80 & 29.40 & 46.90 & PD-L1- \\
\hline 9 & 70 & M & Tongue & T2N0M0 & 1.13 & 59.90 & 1.02 & 0.73 & 38.80 & 4.50 & PD-L1' \\
\hline 10 & 63 & M & Mouth floor & T2N0M0 & 0.81 & 45.00 & 0.73 & 0.55 & 22.40 & 14.80 & PD-L1' \\
\hline 11 & 53 & M & Tongue & T2N0M0 & 1.06 & 74.90 & 0.95 & 0.74 & 80.60 & 84.90 & PD-L1' \\
\hline 12 & 59 & M & Mouth floor & T2N0M0 & 1.19 & 89.80 & 1.07 & 0.80 & 31.40 & 15.80 & PD-L1' \\
\hline 13 & 71 & $\mathrm{~F}$ & Tongue & T2N0M0 & 1.06 & 43.80 & 0.95 & 0.77 & 67.80 & 84.00 & PD-L1 ${ }^{\text {+low }}$ \\
\hline 14 & 41 & M & Tongue & T2N0M0 & 0.98 & 58.10 & 0.88 & 0.55 & 89.00 & 35.60 & PD-L1 ${ }^{\text {+low }}$ \\
\hline 15 & 68 & $\mathrm{~F}$ & Mandibular & T2N0M0 & 1.16 & 44.90 & 1.05 & 1.17 & 56.80 & 75.10 & PD-L1 ${ }^{\text {+low }}$ \\
\hline 16 & 64 & M & Palate & T2N0M0 & 2.10 & 29.00 & 1.89 & 1.12 & 81.40 & 99.00 & PD-L1 ${ }^{\text {+low }}$ \\
\hline 17 & 87 & M & Buccal & T2N0M0 & 1.36 & 72.60 & 1.23 & 0.73 & 37.20 & 39.70 & PD-L1 ${ }^{\text {thigh }}$ \\
\hline 18 & 66 & $\mathrm{~F}$ & Buccal & T2N0M0 & 3.09 & 44.40 & 2.78 & 2.59 & 67.40 & 1.50 & PD-L1 ${ }^{\text {thigh }}$ \\
\hline 19 & 72 & $\mathrm{~F}$ & Mouth floor & T2N2bM0 & 2.62 & 29.20 & 2.36 & 2.42 & 71.00 & 4.70 & PD-L1 ${ }^{\text {thigh }}$ \\
\hline 20 & 83 & $\mathrm{~F}$ & Maxilla & $\mathrm{T} 2 \mathrm{~N} 2 \mathrm{bM} 0$ & 2.13 & 135.10 & 1.92 & 1.28 & 86.40 & 91.40 & PD-L1 ${ }^{\text {+high }}$ \\
\hline 21 & 76 & $\mathrm{~F}$ & Maxilla & T3N0M0 & 0.56 & 101.80 & 0.50 & 0.34 & 30.20 & 93.10 & PD-L1' \\
\hline Mean & 64 & & & & 1.45 & 64.79 & 1.31 & 0.97 & 51.92 & 61.45 & \\
\hline SD & 13 & & & & 0.64 & 27.26 & 0.57 & 0.57 & 24.65 & 34.97 & \\
\hline Median & 65 & & & & 1.19 & 59.90 & 1.07 & 0.80 & 49.25 & 75.10 & \\
\hline
\end{tabular}

DCE-MRI, dynamic contrast-enhanced magnetic resonance imaging; MVD, microvessel density; VEGF, vascular endothelial growth factor; PD-L1; programmed death ligand-1; PD-L1' ${ }^{-}$, negative PD-L1; PD-L1 ${ }^{\text {+low }}$, low-positive PD-L1; PD-L1 ${ }^{\text {thigh }}$, high-positive PD-L1; M, male; $\mathrm{F}$, female; SD, standard deviation.

PD-L1 expression was scored using the semi-quantitative tumor proportion score (TPS), which uses a scale of $0-100 \%$ to define the percentage of tumor cells with membranous and cytoplasmic PD-L1 expression. PD-L1 positivity was predefined using a TPS cutoff of $\geq 1 \%$ and clinically relevant cutoffs of $\geq 1$ and $\geq 50 \%$ were used. Tumor-associated immune cells (macrophages and lymphocytes) were not accounted for by using the TPS. Based on the TPS, the samples were sorted into three groups: Negative $(<1 \%)$, low-positive $(1-49 \%)$ and high-positive (50-100\%) PD-L1 expression.

Assessment of VEGF expression. To quantify VEGF expression, 5 fields were acquired at x200 magnification and 2 areas of 100 tumor cells were quantified in each of the 5 fields. VEGF, expressed as a percentage, was defined as the number of positively stained cells to the total number of cells. The samples were sorted into low and high VEGF expression groups based on the median VEGF expression.

Statistical analysis. Statistical analyses were performed using SPSS v27.0 software (IBM Corp.). The correlation between DCE-MRI parameters (CI-max, T-max, CI-gain and CI-peak) and MVD, PD-L1 and VEGF expression were evaluated using
Spearman's correlation coefficient (r), which uses a scale from -1 to 1 , where 1 implies a perfect positive correlation and -1 indicates a perfect negative correlation. Statistical differences between the DCE-MRI parameters and MVD and PD-L1 expression levels were determined using a U-Mann Whitney test or a Kruskal Wallis test followed by a Dunn's post hoc test. $\mathrm{P}<0.05$ was considered to indicate a statistically significant difference.

\section{Results}

Patients' characteristics. A total of 21 patients with OSCC were analyzed in the present study. The patients' characteristics, DCE-MRI parameters and immunohistochemical findings are detailed in Table I.

DCE-MRI findings. Amongst the OSCC samples, the mean CI-max was found to be $1.45 \pm 0.64$ (range, 0.56-3.09), which occurred at a mean T-max of $65 \mathrm{sec}$. The mean CI-peak was recorded as $1.31 \pm 0.57$ (range, $0.50-2.78$ ) and the mean CI-gain was $0.97 \pm 0.57$ (range, 0.34-2.59; Table I).

Histopathological findings. CD31 staining was performed on all patient samples. Representative images of low and high 

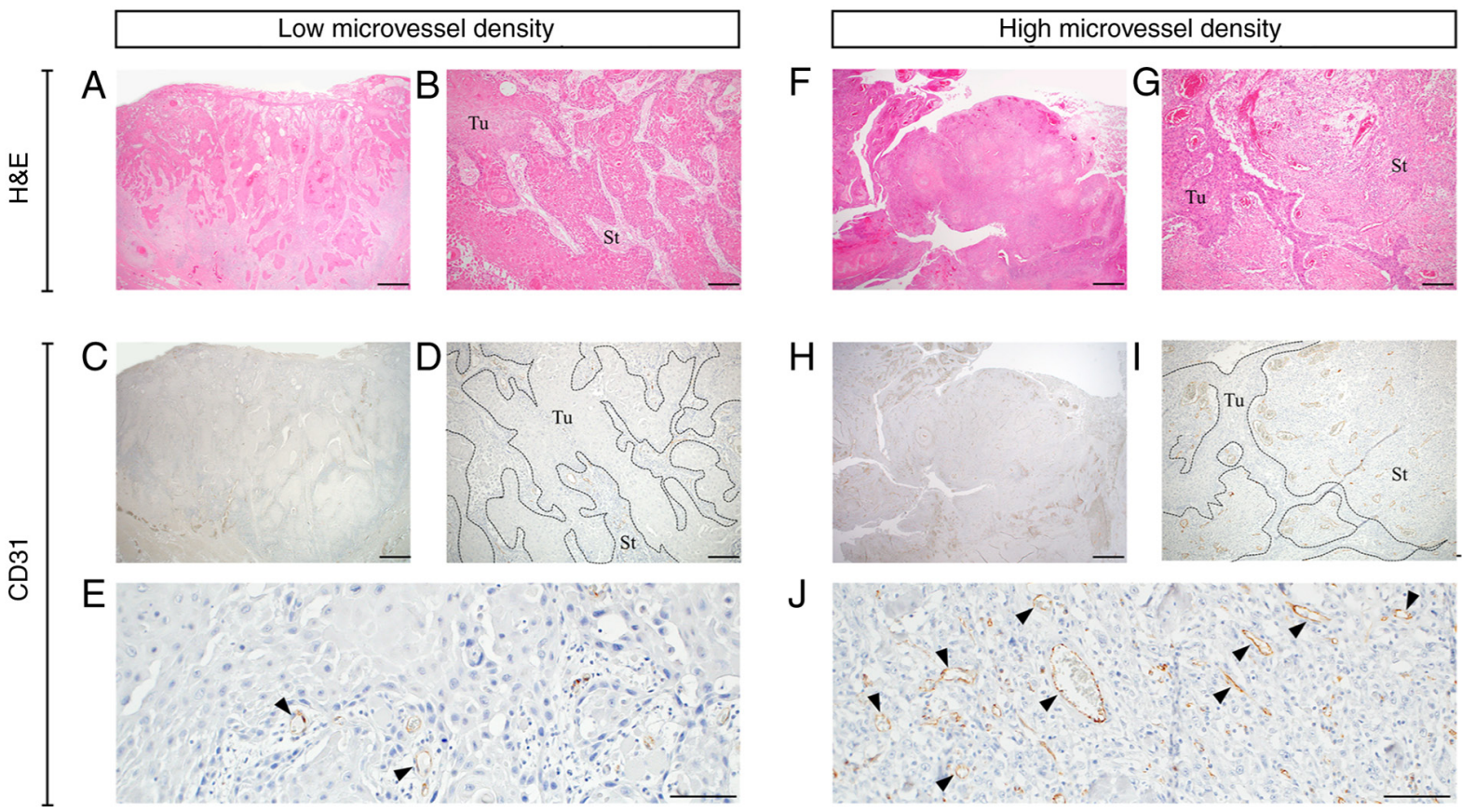

Figure. 2. Microvessel density in OSCC. (A-E) Representative OSCC case with low microvessel density. (F-J) Representative OSCC case with high microvessel density. (A, B, F and G) H\&E staining. (C, D, E, H, I and J) Immunohistochemistry staining for CD31. The slides were examined at low and high magnifications. Borders between Tu and St are shown using the dotted lines. Arrowheads indicate CD31-positive microvessels. Scale bar=1 mm in A, C, F and H. Scale bar $=200 \mu \mathrm{m}$ in B, D, G and I. Scale bar $=100 \mu \mathrm{m}$ in E and J. OSCC, oral squamous cell carcinoma; Tu, tumor; St, stroma; H\&E, hematoxylin and eosin.

MVD staining patterns are presented in Fig. 2. CD31-positive microvessels were observed in the cancer stromal area. Microvessels were either narrow without a lumen or rounded with a lumen. The mean MVD was discovered to be 51.92 \pm 24.65 (range, 5.20-89.00). The median MVD, which was used to divide the patient cohort into low $(\mathrm{n}=10)$ and high (n=11) MVD groups, was 49.25 (Table I).

According to the previously outlined criteria, the patient cohort was divided into negative $(n=9)$, low-positive $(n=6)$ and high-positive ( $n=6)$ PD-L1 expression groups. Representative samples of negative, low-positive and high-positive PD-L1 staining patterns are presented in Fig. 3. The results demonstrated that PD-L1 was expressed in the membrane and cytoplasm of cancer cells. In particular, PD-L1 expression was higher in the cytoplasm of the high-positive PD-L1 expression group compared with that in the other groups. The PD-L1 expression levels in each patient are seen in Table I.

Analysis of DCE-MRI parameters and histopathological findings. The correlations between the DCE-MRI parameters and histopathological findings are shown in Table II. The MVD was found to be negatively correlated with the T-max $(\mathrm{r}=-0.61 ; \mathrm{P}=0.003)$ and positively correlated with the CI-gain $(\mathrm{r}=0.46 ; \mathrm{P}=0.037)$. PD-L1 expression was identified to be positively correlated with the CI-max $(\mathrm{r}=0.57 ; \mathrm{P}=0.007)$, CI-peak $(\mathrm{r}=0.57 ; \mathrm{P}=0.007)$ and $\mathrm{CI}-$ gain $(\mathrm{r}=0.58 ; \mathrm{P}=0.006)$. PD-L1 expression was also discovered to be positively correlated with $\operatorname{MVD}(\mathrm{r}=0.66 ; \mathrm{P}=0.001)$.

The DCE-MRI parameters according to the MVD levels are shown in Table III and Fig. 4A. Comparison of the DCE-MRI parameters according to the MVD levels showed that the mean CI-max, CI-peak and CI-gain were higher in the high MVD tumors, whereas the T-max was longer in the low MVD tumors. The differences in the CI-gain and T-max parameters between the two MVD groups were statistically significant $(\mathrm{P}<0.05$ and $\mathrm{P}<0.01$, respectively).

The DCE-MRI parameters and MVD according to the PD-L1 expression levels are shown in Table III and Fig. 4B. Comparison of the DCE-MRI parameters according to the PD-L1 expression levels showed that the mean CI-max, CI-peak and CI-gain were highest in the tumors with high-positive PD-L1 expression, whereas the mean T-max was longest in the tumors with negative PD-L1 expression. The differences in CI-max, CI-peak and CI-gain among the three PD-L1 expression level groups were statistically significant $(\mathrm{P}<0.05)$, particularly between the tumors with negative and high-positive PD-L1 expression. The mean MVD was also highest in the tumors with high-positive PD-L1 expression, and differences in the mean MVD between the three PD-L1 expression level groups were statistically significant $(\mathrm{P}<0.05)$.

Analysis of VEGF expression. The median VEGF expression, which was used to divide the patient cohort into low $(n=10)$ and high $(\mathrm{n}=11)$ VEGF expression groups, was 75.10 (Table I). Representative samples of low and high VEGF staining patterns are presented in Fig. S1. VEGF expression was not significantly correlated with the DCE-MRI parameters; CI-max $(\mathrm{P}=0.634)$, T-max $(\mathrm{P}=0.946)$, CI-peak $(\mathrm{P}=0.634)$ and CI-gain $(\mathrm{P}=0.839)$. VEGF expression was also not significantly correlated with MVD $(\mathrm{P}=0.302)$ and $\mathrm{PD}-\mathrm{L} 1$ expression $(\mathrm{P}=0.513)$ (Table II).

Tumor recurrence. The association between PD-L1 expression and tumor recurrence was examined. Seventeen patients fulfilled the requirement of 5-year follow-up. The high-positive PD-L1 expression rate in patients with recurrence was 50\%. The 

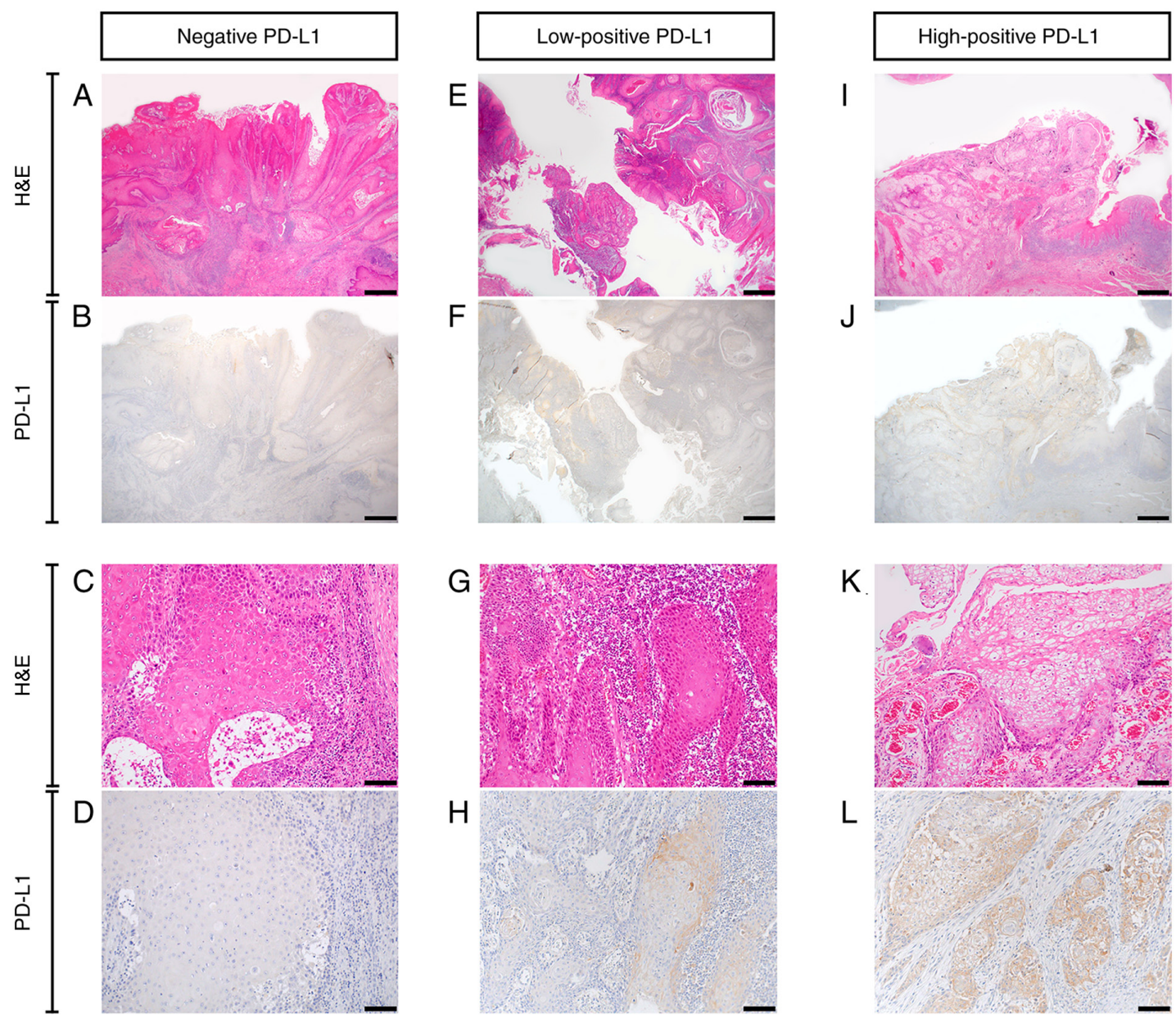

Figure. 3. PD-L1 expression in oral squamous cell carcinoma. (A-D) Negative PD-L1 expression. (E-H) Low-positive PD-L1 expression. (I-L) High-positive PD-L1 expression. (A, C, E, G, I and K) H\&E staining. (B, D, F, H, J and L) Immunohistochemistry staining for PD-L1. Scale bar=1 mm in A, B, E, F, I and J. Scale bar $=100 \mu \mathrm{m}$ in C, D, G, H, K and L. PD-L1, programmed death ligand-1; H\&E, hematoxylin and eosin.

recurrence rate in patients with high-positive PD-L1 expression was $80 \%$. However, the association was not statistically significant ( $\mathrm{P}=0.165$ via Fisher's exact test) (Table $\mathrm{SI})$.

\section{Discussion}

The present study aimed to investigate the correlations between DCE-MRI parameters and histopathological parameters in patients with OSCC. The results demonstrated significant correlations among various DCE-MRI parameters, MVD and PD-L1 expression.

The implementation of functional imaging modalities, including DCE-MRI, in clinical practice has led to an increased number of studies investigating the potential of these modalities in the assessment of histopathological parameters. DCE-MRI reflects tissue perfusion and vascularization and is presumed to be based on tissue composition parameters, such as cellularity and vascular density (18). Previous studies have investigated the findings obtained from DCE-MRI in various types of cancer; however, only a few studies have to date assessed the correlation between DCE-MRI findings and the histopathological parameters in HNSCC and OSCC in particular (9,18-20).

Surov et al $(18,19)$ and Jansen et al $(20)$ evaluated DCE-MRI perfusion and DCE-MRI histogram-based parameters in HNSCC. The results identified correlations between DCE-MRI parameters and histopathological findings reflecting VEGF and Ki-67 expression, MVD, cellularity and nucleic content. The results from these studies suggested that DCE-MRI may be used to assess histopathological parameters in patients with HNSCC.

In the present study, quantitative DCE-MRI parameters were derived from the SI and CI curves. Previous studies have reported the value of these SI- and CI-based parameters in the identification of oral lesion characteristics, which can contribute to the diagnosis of oral lesions (21-25). In a previous study evaluating quantitative DCE-MRI CI-based parameters in patients with OSCC, Unetsubo et al (9) suggested that DCE-MRI may be useful for the assessment of MVD, as a significant correlation was identified between CI-gain and MVD. It is important to note that the present study was conducted using 3T MRI and analyzed CD31 expression via IHC, whereas Unetsubo et al (9) used 1.5T MRI and analyzed CD34 expression.

To interpret the MVD findings from the present study, the study population was categorized into low and high MVD groups based on the median MVD value. The results revealed that MVD was negatively correlated with the T-max and positively correlated with the CI-gain. These paradoxical observations in the correlations could be explained by the 
Table II. Correlation between DCE-MRI parameters and immunohistochemical findings.

\begin{tabular}{lcrr}
\hline Variables & MVD $(\mathrm{n}=21)$ & PD-L1 $(\mathrm{n}=21)$ & VEGF $(\mathrm{n}=21)$ \\
\hline DCE-MRI parameters & & & \\
CI-max & $0.20(\mathrm{P}=0.373)$ & $0.57(\mathrm{P}=0.007)^{\mathrm{a}}$ & $-0.11(\mathrm{P}=0.634)$ \\
T-max & $-0.61(\mathrm{P}=0.003)^{\mathrm{a}}$ & $-0.42(\mathrm{P}=0.055)$ & $-0.02(\mathrm{P}=0.946)$ \\
CI-peak & $0.20(\mathrm{P}=0.373)$ & $0.57(\mathrm{P}=0.007)^{\mathrm{a}}$ & $-0.11(\mathrm{P}=0.634)$ \\
CI-gain & $0.46(\mathrm{P}=0.037)^{\mathrm{a}}$ & $0.58(\mathrm{P}=0.006)^{\mathrm{a}}$ & $-0.05(\mathrm{P}=0.839)$ \\
IHC staining & - & $0.66(\mathrm{P}=0.001)^{\mathrm{a}}$ & $0.24(\mathrm{P}=0.302)$ \\
MVD & - & - & $-0.15(\mathrm{P}=0.513)$ \\
PD-L1 & $0.66(\mathrm{P}=0.001)^{\mathrm{a}}$ & & \\
\hline
\end{tabular}

Correlation given by Spearman-Rho test. ${ }^{a} \mathrm{P}<0.05$. DCE-MRI, dynamic contrast-enhanced magnetic resonance imaging; MVD, microvessel density; PD-L1, programmed death ligand-1; VEGF, vascular endothelial growth factor; IHC, immunohistochemistry.

Table III. DCE-MRI parameters and immunohistochemical findings according to the microvessel density and PD-L1 expression.

\begin{tabular}{|c|c|c|c|c|c|c|c|c|}
\hline Variables & $\begin{array}{l}\text { All patients } \\
\quad(n=21)\end{array}$ & $\begin{array}{l}\text { MVD, low } \\
(n=10)\end{array}$ & $\begin{array}{l}\text { MVD, high } \\
\quad(n=11)\end{array}$ & P-value ${ }^{a}$ & $\begin{array}{l}\text { PD-L1' } \\
(n=9)\end{array}$ & $\begin{array}{c}\text { PD-L1 }{ }^{\text {+low }}, \\
(n=6)\end{array}$ & $\begin{array}{c}\text { PD-L1 }{ }^{\text {thigh }}, \\
(n=6)\end{array}$ & P-value ${ }^{b}$ \\
\hline \multicolumn{9}{|c|}{ DCE-MRI parameters } \\
\hline CI-max & 1.45 & 1.18 & 1.70 & $\mathrm{P}=0.38$ & 1.10 & 1.32 & 2.11 & $\mathrm{P}<0.05\left(\mathrm{P}<0.05^{\mathrm{c}}\right)$ \\
\hline T-max & 64.79 & 76.85 & 53.82 & $\mathrm{P}<0.01$ & 75.66 & 51.55 & 61.72 & $\mathrm{P}=0.07$ \\
\hline CI-peak & 1.31 & 1.06 & 1.53 & $\mathrm{P}=0.38$ & 0.99 & 1.19 & 1.90 & $\mathrm{P}<0.05\left(\mathrm{P}<0.05^{\mathrm{c}}\right)$ \\
\hline CI-gain & 0.97 & 0.71 & 1.22 & $\mathrm{P}<0.05$ & 0.70 & 0.83 & 1.53 & $\mathrm{P}<0.05\left(\mathrm{P}<0.05^{\mathrm{c}}\right)$ \\
\hline \multicolumn{9}{|c|}{ IHC staining } \\
\hline MVD & 51.92 & - & - & & 34.67 & 61.51 & 68.20 & $\mathrm{P}<0.05\left(\mathrm{P}<0.05^{\mathrm{c}}\right)$ \\
\hline
\end{tabular}

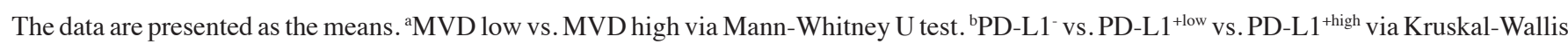
test; ${ }^{\text {PDD-L1 }}{ }^{-}$vs. PD-L1 ${ }^{\text {thigh }}$ via Dunn's test. P $<0.05$ was considered statistically significant. DCE-MRI, dynamic contrast-enhanced magnetic resonance imaging; MVD, microvessel density, PD-L1, programmed death ligand-1; PD-L1', negative PD-L1; PD-L1 ${ }^{\text {+low }}$, low-positive PD-L1; PD-L1 $1^{\text {thigh }}$, high-positive PD-L1; IHC, immunohistochemistry.

nature of the CI-curve. For instance, the correlation between the high MVD and short T-max suggests that in the presence of high blood flow, the contrast agent is rapidly circulated through the blood and absorbed; therefore, the time at which the contrast enhancement reaches its maximum amplitude is short. Conversely, the positive correlation between the high MVD and high CI-gain indicates that in the presence of high blood flow, the gradient on the upslope phase of the enhancement curve is high. Furthermore, the findings from the present study supported the hypothesis that DCE-MRI may be used to assess MVD and be able to distinguish tumors with low and high levels of angiogenesis (18). In fact, the comparison of MRI parameters according to the MVD revealed that the mean CI-gain was significantly increased, while the mean T-max was significantly shorter in high-MVD tumors, suggesting that tumors with high levels of angiogenesis exhibit a higher and faster uptake of the contrast agent. These findings were consistent with a previous study reporting that intratumor angiogenesis can influence the enhanced patterns on the DCE-MRI scan (9). DCE-MRI may therefore represent a promising non-invasive method for assessing angiogenesis in patients with OSCC.
The present study also evaluated VEGF expression. The correlations between DCE-MRI parameters and VEGF expression were found to be not statistically significant. The correlation between MVD and VEGF expression was also found to be not statistically significant, although MVD and VEGF expression exhibited similar tendency. Well-defined markers of angiogenesis include microvessels stained with CD31 and VEGF (26). However, more than one factor is required to suggest an angiogenic response. Vessel maturation is a crucial factor for angiogenesis regulation (27). An indicative of vessel maturation is the presence of pericytes and of smooth muscle cells stained by $\alpha$-smooth muscle actin ( $\alpha$-SMA) $(27,28)$. VEGF is a positive regulator of pericyte function that has a potential role in the maintenance of mature blood vessels $(28,29)$. However, CD31 is expressed in both mature and immature blood vessels (28). These differences in expression between angiogenesis markers may explain the differences observed in correlations with DCE-MRI parameters. Thus, a more thorough study may be required to further clarify the association between DCE-MRI and angiogenesis using different markers (CD31, VEGF and $\alpha$-SMA). 
A

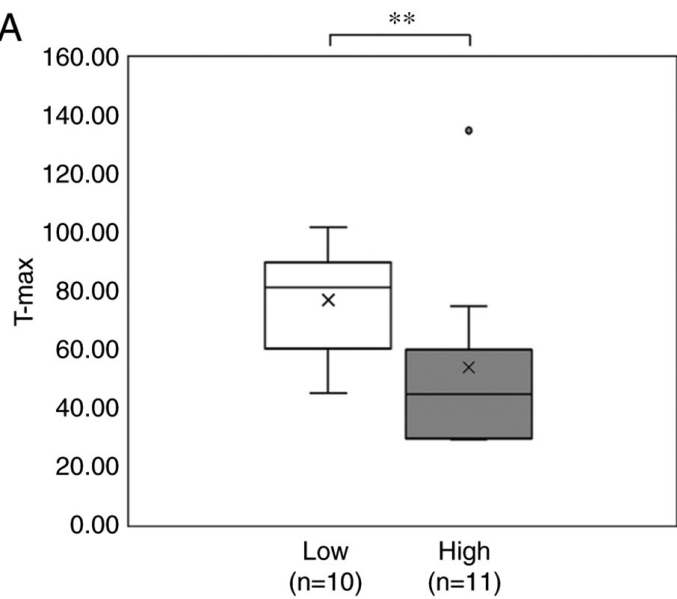

B
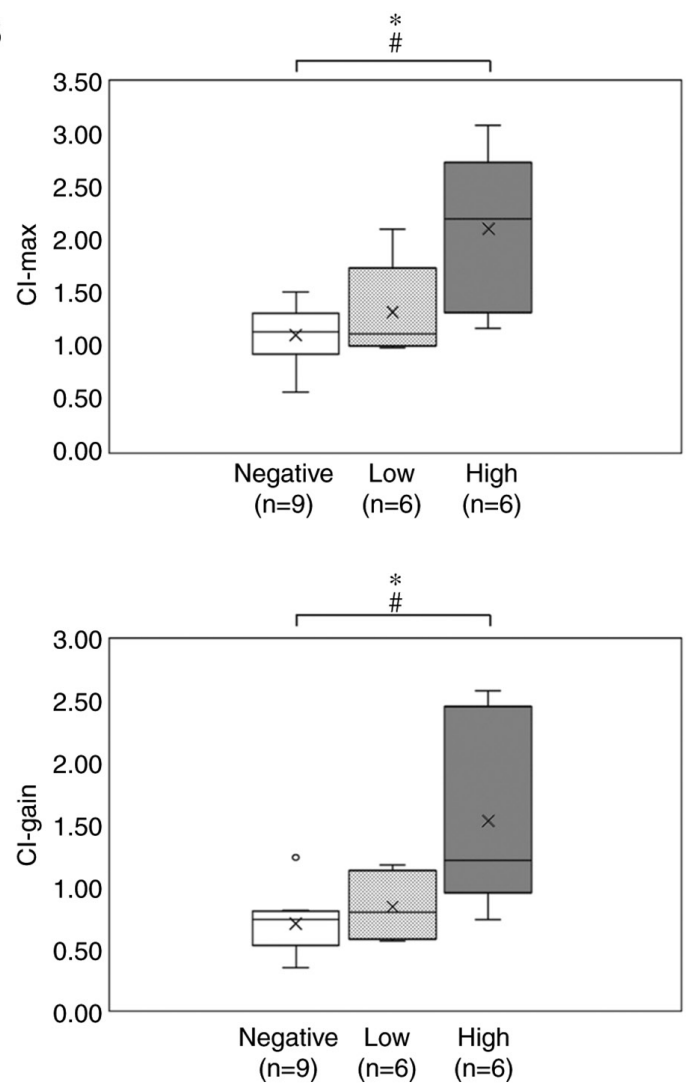
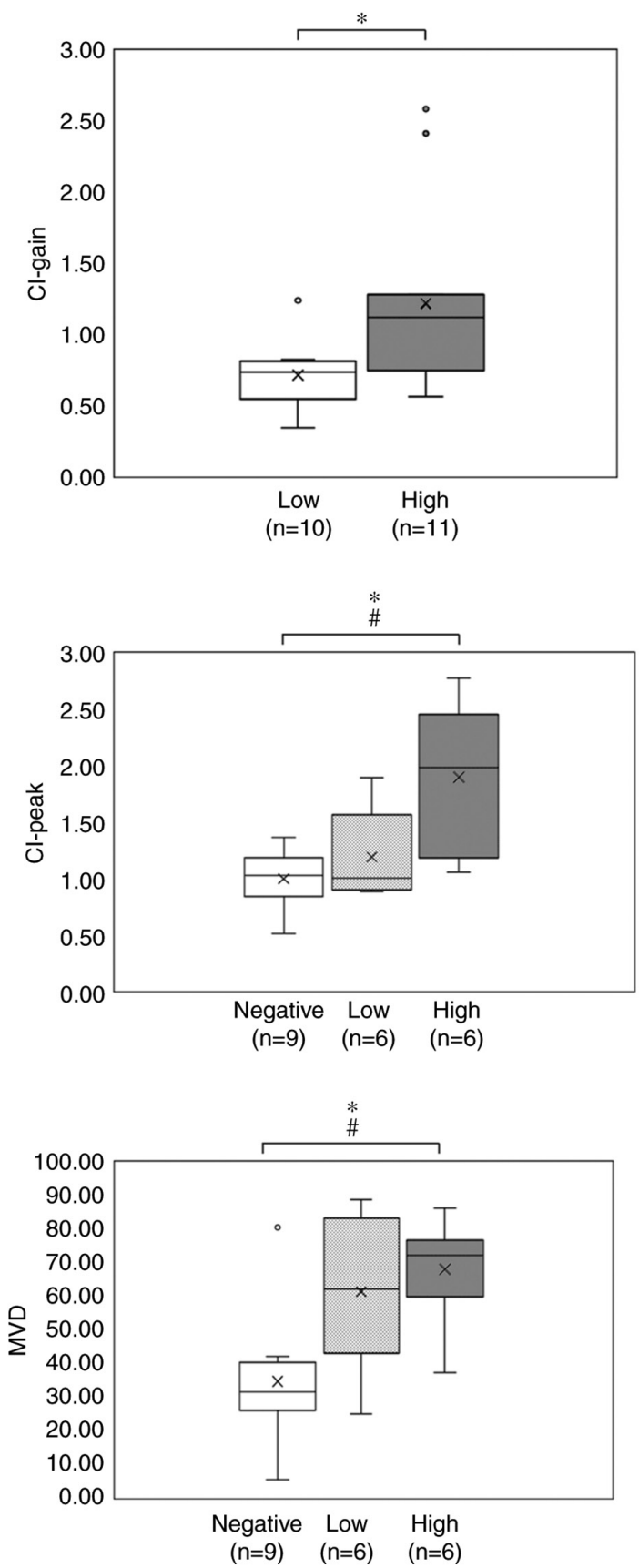

Figure. 4. DCE-MRI parameters and histopathological findings. (A) DCE-MRI parameters according to the MVD levels. *P<0.05, ${ }^{* *} \mathrm{P}<0.01$ via $\mathrm{U}-\mathrm{Mann}$ Whitney test. (B) DCE-MRI parameters and MVD according to the PD-L1 expression levels. "P<0.05 via Kruskal-Wallis test; ${ }^{*} \mathrm{P}<0.05$ via Dunn's test. PD-L1, programmed death ligand-1; DCE-MRI, dynamic contrast-enhanced magnetic resonance imaging; CI, contrast index; MVD, microvessel density.

Highly promising immunotherapies have recently emerged, of which the most prominent subtype is ICIs that target the PD-1/PD-L1 signaling pathway. The binding of PD-1 to its major ligands, PD-L1 and PD-L2, releases inhibitory cytokines that inhibit T-cell activation and proliferation $(8,30)$. PD-1/PD-L1-targeting ICIs disrupt this interaction and allow for immune system recognition, activation and destruction of tumor cells (8).

The first immunotherapy approved for use in HNSCC was granted in 2016. Several ICIs have since been trialed, of which the two most extensively studied are pembrolizumab and nivolumab, which were found to induce considerable and durable responses (1). PD-L1 expression is often used as a prognostic and predictive marker of the response to PD-1/PD-L1 ICIs. Previous evidence has shown that PD-L1 positivity is associated with clinical benefits and improved responses and outcomes (8). Furthermore, high-positive PD-L1 expression is a poor prognostic marker in OSCC (30). Due to the reported correlations between DCE-MRI parameters and MVD and between PD-L1 and MVD $(9,11)$, the present study investigated the potential correlations between DCE-MRI parameters and PD-L1 expression.

A previous study reported that $\mathrm{PD}-\mathrm{L} 1$ expression is upregulated in $18-96 \%$ of OSCC cases (5). This high variability in expression is due to numerous factors, such as intratumor and intertumor heterogeneity, differences in study populations and 
differences in methodology (including different assays used for determining PD-L1 expression, different scoring systems and different cutoff values for positive expression) $(4,8)$. As the TPS reflects PD-L1 expression on cancer cells themselves, but not that of immune cells, this scoring method was used in the present study, with a TPS of $1 \%$ selected as the positivity cutoff value. Using these parameters, the present study demonstrated that PD-L1 was upregulated in $57.1 \%$ of OSCC cases.

In a recent preliminary study, Meyer et al (31) investigated the correlation between DCE-MRI histogram-based parameters and PD-L1 expression. The results identified some correlations between the histogram parameters, the immune cell score and the combined cell score, but not with the TPS (31). However, by using DCE-MRI SI- and CI-based parameters, the present study identified correlations with PD-L1 expression using the TPS. The CI-max, CI-peak and CI-gain were found to be positively correlated with PD-L1 expression. These findings suggested that the high and rapid uptake of the contrast agent may reflect the high levels of PD-L1 expression, thus confirming the hypothesis that PD-L1 expression may be actually reflected in the CI curve.

To further understand the correlations between DCE-MRI parameters and PD-L1 expression, the present study analyzed the DCE-MRI parameters in three different PD-L1 expression groups. The tumors with high-positive PD-L1 expression showed the highest mean CI-max, CI-peak and CI-gain, whereas tumors with negative PD-L1 expression showed the longest T-max. These findings suggested that a higher number of tumor cells expressing PD-L1 may increase the likelihood of the $\mathrm{CI}$ curve to show a high and rapid uptake pattern. In particular, significant differences in these parameters were found between the tumors with negative and high-positive PD-L1 expression. These findings indicated that DCE-MRI SI- and CI-based parameters may be useful for distinguishing tumors with negative PD-L1 expression from tumors with high-positive PD-L1 expression. In addition, the results from the present study revealed that MVD and PD-L1 expression were strongly positively correlated in OSCC. Similar to the DCE-MRI findings, tumors with high-positive PD-L1 expression showed the highest mean MVD among the three expression level groups. Current evidence of the association between PD-L1 expression and MVD has not been well documented and previous reports have produced conflicting results. For example, Yugawa et al (32) demonstrated the absence of correlation between MVD and PD-L1 expression in cancer cells using a positivity cutoff of $1 \%$. Franz et al (33) reported that PD-L1 expression using the combined positive score is negatively correlated with MVD in patients with laryngeal carcinoma. However, Koh et al (11) demonstrated that PD-L1-positive tumors are positively correlated with hypoxia-inducible factors signaling pathway including MVD in patients with classical Hodgkin lymphoma. The present study reported similar findings to the latter, indicating that tumors with high levels of angiogenesis may tend to contain a higher number of tumor cells expressing PD-L1. The detailed mechanism underlying the interaction between angiogenesis and PD-1/PD-L1 signaling pathways should therefore be further investigated in future studies.

The results from the present study were consistent with the findings from previous studies demonstrating that DCE-MRI might be able to predict the response to anti-angiogenic therapy (18). Furthermore, the present findings indicated that using DCE-MRI to identify tumors with high-positive PD-L1 expression (which seemingly have a more favorable response to ICIs) may predict the therapeutic effect and outcome of such immunotherapies. DCE-MRI may also help improving patient selection by identifying patients who are most likely to benefit from the treatment.

The results from the present study were preliminary and further studies should be performed on larger patient populations to validate these findings. Furthermore, configuration of the ROI for T4 tumors has proven to be difficult. Therefore, DCE-MRI assessment of PD-L1 expression may only be beneficial for T1, T2 and T3 tumors according to the TNM classification. Finally, although PD-L1 has shown clinical value as a biomarker, it does not fully predict the response to ICIs. Thus, the assessment of PD-L1 expression using DCE-MRI should solely be performed as an adjunctive examination.

In summary, the findings from the present study indicated that the CI parameters derived from DCE-MRI may be associated with PD-L1 expression on tumor cells. A high and rapid uptake pattern of the CI curve was found to be significantly correlated with high-positive PD-L1 expression. Therefore, these findings suggested that DCE-MRI may be considered as a valuable non-invasive method for assessing PD-L1 expression and the therapeutic efficacy of ICIs in patients with OSCC.

\section{Acknowledgements}

The authors would like to thank Dr Angela Morben for editing a draft of this manuscript.

\section{Funding}

The present study was supported by a JSPS Grant-in-Aid for Young Scientists (grant no. JP 18K17169). The funding source had no role in the study design, analysis, writing or submission of the data.

\section{Availability of data and materials}

The datasets used and/or analyzed during the current study are available from the corresponding author on reasonable request.

\section{Authors' contributions}

NT, MF, JIA, YY, TK and MH conceived the idea for the study. NT, MF, TO and HK designed the study. NT, MF, HK, MWO, SO and YT performed the experiments. NT, MF, TO and HK were responsible for the quality control of data and algorithms. NT, MF, TO, HK and MB analyzed and interpreted the data. NT, MF and TO performed the statistical analysis. MF and HK confirmed the authenticity of all the raw data. NT, SO, YT and $\mathrm{MB}$ wrote the initial draft of the manuscript. MF, TO and HK edited the manuscript. JIA, HN, TK and MH reviewed the manuscript. All authors have read and approved the final manuscript.

\section{Ethics approval and consent to participate}

The study protocol was approved by the institutional review board of Okayama University Graduate School of Medicine, 
Dentistry and Pharmaceutical Sciences, Okayama University Hospital, Ethics Committee (approval no. 1807-008).

\section{Patient consent for publication}

Not applicable.

\section{Competing interests}

The authors declare that they have no competing interests.

\section{References}

1. Bauml JM, Aggarwal C and Cohen RB: Immunotherapy for head and neck cancer: Where are we now and where are we going? Ann Transl Med 7 (Suppl 7): S75, 2019.

2. Twomey JD and Zhang B: Cancer immunotherapy update: FDA-approved checkpoint inhibitors and companion diagnostics. AAPS J 23: 39, 2021

3. Samra B, Tam E, Baseri B and Shapira I: Checkpoint inhibitors in head and neck cancer: Current knowledge and perspectives. J Investig Med 66: 1023-1030, 2018

4. Cohen EEW, Bell RB, Bifulco CB, Burtness B, Gillison ML Harrington KJ, Le QT, Lee NY, Leidner R, Lewis RL, et al: The society for immunotherapy of cancer consensus statement on immunotherapy for the treatment of squamous cell carcinoma of the head and neck (HNSCC). J Immunother Cancer 7: 184, 2019.

5. Lenouvel D, González-Moles MÁ, Talbaoui A, Ramos-García P, González-Ruiz L, Ruiz-Ávila I and Gil-Montoya JA: An update of knowledge on PD-L1 in head and neck cancers: Physiologic, prognostic and therapeutic perspectives. Oral Dis 26: 511-526, 2020.

6. De Vicente JC, Rodríguez-Santamarta T, Rodrigo JP, Blanco-Lorenzo V, Allonca E and García-Pedrero JM: PD-L1 expression in tumor cells is an independent unfavorable prognostic factor in oral squamous cell carcinoma. Cancer Epidemio Biomarkers Prev 28: 546-554, 2019.

7. Forster MD and Devlin MJ: Immune checkpoint inhibition in head and neck cancer. Front Oncol 8: 310, 2018.

8. McCusker MG, Orkoulas-Razis D and Mehra R: Potential of pembrolizumab in metastatic or recurrent head and neck cancer: Evidence to date. Onco Targets Ther 13: 3047-3059, 2020.

9. Unetsubo T, Konouchi H, Yanagi Y, Murakami J, Fujii M, Matsuzaki H, Hisatomi M, Nagatsuka H and Asaumi JI: Dynamic contrast-enhanced magnetic resonance imaging for estimating tumor proliferation and microvessel density of oral squamous cell carcinomas. Oral Oncol 45: 621-626, 2009.

10. Pałasz P, Adamski Ł, Górska-Chrząstek M, Starzyńska A and Studniarek M: Contemporary diagnostic imaging of oral squamous cell carcinoma-A review of literature. Pol J Radiol 82: 193-202, 2017.

11. Koh YW, Han JH, Yoon DH, Suh C and Huh J: PD-L1 expression correlates with VEGF and microvessel density in patient with uniformly treated classical Hodgkin lymphoma. Ann Hematol 96: 1883-1890, 2017.

12. Szafarowski T, Sierdzinski J, Szczepanski MJ, Whiteside TL, Ludwig N and Krzeski A: Microvessel density in head and neck squamous cell carcinoma. Eur Arch Otorhinolaryngol 275: 1845-1851, 2018.

13. Brierley JD, Gospodarowicz MK and Wittekind CH (eds): TNM Classification of Malignant Tumours, 8th Edition. Oxford, UK: Hoboken, NJ: John Wiley \& Sons, Inc.; 2017.

14. Takabatake K, Tsujigiwa H, Nakano K, Inada Y, Qiusheng S, Kawai H, Sukegawa S, Fushimi S and Nagatsuka H: Geometrical structure of honeycomb TCP to control dental pulp-derived cell differentiation. Materials (Basel) 13: 5155, 2020

15. Nowak-Sliwinska P, Alitalo K, Allen E, Anisimov A, Aplin AC, Auerbach R, Augustin HG, Bates DO, van Beijnum JR, Bender RHF, et al: Consensus guidelines for the use and interpretation of angiogenesis assays. Angiogenesis 21:425-532, 2018

16. Weidner N, Semple JP, Welch WR and Folkman J: Tumor angiogenesis and metastasis-correlation in invasive breast carcinoma. N Engl J Med 324: 1-8, 1991.

17. Fang L, He Y, Liu Y, Ding H, Tong Y, Hu L, Wang C, Zhang Y, Zheng $\mathrm{X}$ and Huang P: Adjustment of microvessel area by stromal area to improve survival prediction in non small cell lung cancer. J Cancer 10: 3397-3406, 2019.
18. Surov A, Meyer HJ, Gawlitza M, Höhn AK, Boehm A, Kahn T and Stumpp P: Correlations between DCE MRI and histopathological parameters in head and neck squamous cell carcinoma. Transl Oncol 10: 17-21, 2017.

19. Surov A, Meyer HJ, Leifels L, Höhn AK, Richter C and Winter K: Histogram analysis parameters of dynamic contrast-enhanced magnetic resonance imaging can predict histopathological findings including proliferation potential, cellularity, and nucleic areas in head and neck squamous cell carcinoma. Oncotarget 9: 21070-21077, 2018

20. Jansen JFA, Carlson DL, Lu Y, Stambuk HE, Moreira AL, Singh B, Patel SG, Kraus DH, Wong RJ, Shaha AR, et al: Correlation of a priori DCE-MRI and 1H-MRS data with molecular markers in neck nodal metastases: Initial analysis. Oral Oncol 48: 717-722, 2012.

21. Asaumi J, Yanagi Y, Konouchi H, Hisatomi M, Matsuzaki H and Kishi K: Application of dynamic contrast-enhanced MRI to differentiate malignant lymphoma from squamous cell carcinoma in the head and neck. Oral Oncol 40: 579-584, 2004.

22. Yanagi Y, Asaumi JI, Unetsubo T, Ashida M, Takenobu T, Hisatomi M, Matsuzaki H, Konouchi H, Katase $\mathrm{N}$ and Nagatsuka H: Usefulness of MRI and dynamic contrast-enhanced MRI for differential diagnosis of simple bone cysts from true cysts in the jaw. Oral Surg Oral Med Oral Pathol Oral Radiol Endod 110: 364-369, 2010.

23. Hisatomi M, Yanagi Y, Konouchi H, Matsuzaki H, Takenobu T, Unetsubo T and Asaumi JI: Diagnostic value of dynamic contrast-enhanced MRI for unilocular cystic-type ameloblastomas with homogeneously bright high signal intensity on T2-weighted or STIR MR images. Oral Oncol 47: 147-152, 2011.

24. Matsuzaki H, Hara M, Yanagi Y, Asaumi JI, Katase N, Unetsubo T, Hisatomi M, Konouchi H, Takenobu T and Nagatsuka H: Magnetic resonance imaging (MRI) and dynamic MRI evaluation of extranodal non-Hodgkin lymphoma in oral and maxillofacial regions. Oral Surg Oral Med Oral Pathol Oral Radiol 113: 126-133, 2012.

25. Fujita M, Matsuzaki H, Yanagi Y, Hara M, Katase N, Hisatomi M, Unetsubo T, Konouchi H, Nagatsuka H and Asaumi JI: Diagnostic value of MRI for odontogenic tumours. Dentomaxillofac Radiol 42: 20120265, 2013.

26. Schlüter A, Weller P, Kanaan O, Nel I, Heusgen L, Höing B, Haßkamp P, Zander S, Mandapathil M, Dominas N, et al: CD31 and VEGF are prognostic biomarkers in early-stage, but not in late-stage, laryngeal squamous cell carcinoma. BMC Cancer 18: 272,2018

27. Tonino $\mathrm{P}$ and Abreu $\mathrm{C}$ : Microvessel density is associated with VEGF and $\alpha$-SMA expression in different regions of human gastrointestinal carcinomas. Cancers (Basel) 3: 3405-3418, 2011.

28. Usuda K, Iwai S, Funasaki A, Sekimura A, Motono N, Ueda Y, Shimazaki M and Uramoto H: Expression and prognostic impact of VEGF, CD31 and $\alpha$ SMA in resected primary lung cancers. Anticancer Res 38: 4057-4063, 2018.

29. dela Paz NG, Walshe TE, Leach LL, Saint-Geniez M and D'Amore PA: Role of shear-stress-induced VEGF expression in endothelial cell survival. J Cell Sci 125: 831-843, 2012.

30. He J, Chen XF, Xu MG and Zhao J: Relationship of programmed death ligand-1 expression with clinicopathological features and prognosis in patients with oral squamous cell carcinoma: A meta-analysis. Arch Oral Biol 114: 104717, 2020.

31. Meyer HJ, Höhn AK and Surov A: Associations between histogram analysis parameters derived from dynamic-contrast enhanced MRI and PD L1-expression in head and neck squamous cell carcinomas. A preliminary study. Magn Reson Imaging 72: 117-121, 2020.

32. Yugawa $\mathrm{K}$, Itoh $\mathrm{S}$, Yoshizumi $\mathrm{T}$, Iseda N, Tomiyama T, Toshima $\mathrm{T}$ Harada N, Kohashi K, Oda Y and Mori M: Prognostic impact of tumor microvessels in intrahepatic cholangiocarcinoma: Association with tumor-infiltrating lymphocytes. Mod Pathol 34: 798-807, 2021.

33. Franz L, Alessandrini L, Calvanese L, Crosetta G, Frigo AC and Marioni G: Angiogenesis, programmed death ligand 1 (PD-L1) and immune microenvironment association in laryngeal carcinoma. Pathology: May 13, 2021 (Epub ahead of print).

This work is licensed under a Creative Commons Attribution-NonCommercial-NoDerivatives 4.0 International (CC BY-NC-ND 4.0) License. 